\title{
Study of single mode tapered fiber-optic interferometer of different waist diameters and its application as a temperature sensor
}

T. K. Yadav

\section{A. Mustapa}

M. H. Abu Bakar

M. A. Mahdi

mam@upm.edu.my

\begin{abstract}
Wireless and Photonic Networks Research Center, Faculty of Engineering, Universiti Putra Malaysia, 43400 UPM Serdang, Selangor, Malaysia

Wireless and Photonic Networks Research Center, Faculty of Engineering, Universiti Putra Malaysia, 43400 UPM Serdang, Selangor, Malaysia

Wireless and Photonic Networks Research Center, Faculty of Engineering, Universiti Putra Malaysia, 43400 UPM Serdang, Selangor, Malaysia

Wireless and Photonic Networks Research Center, Faculty of Engineering, Universiti Putra Malaysia, 43400 UPM Serdang, Selangor, Malaysia
\end{abstract}

We have proposed a study on single-mode tapered optical fiber for temperature sensing application. A theoretical analysis and its experimental validation were carried out to study the taper profile for highly sensitive temperature sensor. Experiments were performed to observe a wavelength shift of transmission spectra with different taper profiles. The effects of taper profiles on the sensitivity of the sensor were also investigated. Our results indicate that the tapered fiber-based temperature sensor has sensitivity in the range of 0.01143 to $0.03406 \mathrm{~nm} /{ }^{\circ} \mathrm{C}$. The findings also demonstrate that the sensor sensitivity can be adjusted with variation to the taper profile.

[DOI: http://dx.doi.org/10.2971/jeos.2014.14024]

Keywords: Tapered optical fiber, fiber sensor, temperature sensor

\section{INTRODUCTION}

Optical fiber has been widely studied owing to its potential application not only in communication but also in instrumentation, laser, nonlinear devices and sensing [1]-[4]. A number of optical fiber temperature sensors have been developed so far based on fiber Bragg gratings [5], long period gratings [6, 7], and photonic crystal fiber [8,9] because of its light weight, accuracy, compact size and immunity to electromagnetic, radio-frequency, and microwave interferences [10]-[14]. More recently, single-mode tapered fibers (SMTFs) have attracted great attention from researchers as an alternative for a variety of sensors. In general, SMTF-based sensors are more sensitive than typical cladding-removed fiber sensors [15]. Moreover, they are more compact and simple to make. Most of the SMTF-based sensors reported so far employ non-adiabatic biconically tapered fibers [10]-[14]. The modeling and fabrication of such sensors have been discussed at great length by several authors [12, 13].

SMTF is fabricated by reducing the core diameter via heating a section of the fiber while pulling both of its ends, creating biconical transition regions with a relatively long waist region. The waist diameter can be made to be only a few microns over a length of a few centimeters. Under these conditions, the original fiber core becomes so small that it has no significant influence any more and the light is then guided solely by the airglass interface. If the taper angle is large, the fundamental core mode couples power to one or more cladding modes guided by the cladding air interface due to the large refractive index difference between air and glass. If we assume that the taper is axially symmetric, then the fundamental mode $\mathrm{LP}_{01}$ can couple only to the higher order modes with the same azimuthal symmetry that is $\mathrm{LP}_{0 \mathrm{~m}}$ modes. The interference between these modes results in oscillations in the output spectrum. A number of sensors using conventional single mode fibers utilizing interferometry have been reported $[16,17]$. In particular, the single mode tapered fiber interferometer-based sensors have the advantages of compact structure, good stability, and easy fabrication [18]. The change of the ambient temperature can significantly change the indices of propagating modes and length of the tapered fiber, consequently changing the phase shift of the interference fringe pattern. Thus, the change in temperature can be measured by the shift in the interference fringe pattern and allows tapered fiber to be used as a temperature sensor.

In the present work, our focus is to study the effect of variation to the waist diameter of a tapered single mode optical fiber functioning as a temperature sensor. In this sensor, spectrum of the transmitted light is measured and the effect of temperature change is observed as a spectral shift of the transmission dips associated with the coupled modes. The measurement of this wavelength shift due to change in temperature is the basis of SMTF temperature sensor. To optimize the best geometry of the proposed device, we have studied six tapered single-mode optical fiber of different waist diameter i.e., 12, 10, 9, 8 and $7 \mu \mathrm{m}$ for one taper profile. In our proposed device, the shift in the pattern strongly depends on the temperature changes. The temperature range in this study is between $30^{\circ} \mathrm{C}$ to $150^{\circ} \mathrm{C}$. 


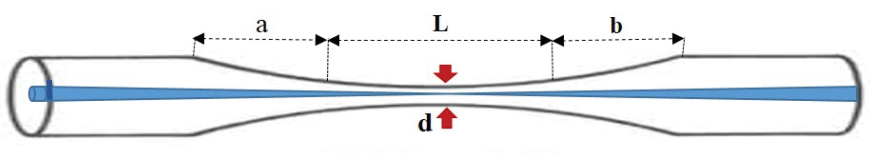

Waist Length (L)

FIG. 1 Schematic diagram of single mode tapered fiber, which is used as the sensor. The downtaper and uptaper regions, $a$ and $b$, are the transition regions where the coupling and recombination of modes occur. The waist diameter is $d$ and $L$ is the waist length.

\section{THEORETICAL DESCRIPTIONS}

When the fundamental core mode propagates in single mode optical fiber from untapered region to tapered region, it excites the higher order modes in the tapered region. It is clear from the geometry of the tapered fiber as shown in Figure 1 that the fundamental modes and higher order modes are coupled together in the untapered region, forming interferometric pattern (Mach-Zehnder interferometer) due to large difference in indices of air and glass and the waist length corresponding to the physical length of the interferometer.

To further understand this coupling phenomenon, let us assume that only two modes exist in the uncollapsed section of the SMF and the effective indices of these modes are $n_{1}$ and $n_{2}$. Thus, the resultant intensity at the end of the SMF is [19]

$$
I_{T}=I_{c o}+I_{c l}+2 \sqrt{I_{c o} I_{c l}} \cos \phi,
$$

where $I_{c o}$ and $I_{c l}$ are the intensities of the two interfering modes and $\phi$ is the phase difference between the two interfering modes, i.e. the phase of the resultant interferometric intensity pattern. Since only one cladding mode is excited, we will have an interferometer whose physical length (waist length $L$ as shown in Figure 1) is exactly the same but with different optical lengths to each other due to the difference in indices of core and cladding. Multiplying it with the difference in indices $\left(\Delta n=n_{1}-n_{2}\right)$ of each excited mode gives the optical path difference of the two interfering modes. Thus the relative phase difference between the two interfering modes can be described by

$$
\phi=\frac{2 \pi}{\lambda} \Delta n L,
$$

where $\lambda$ is the central wavelength of the light source. Since the refractive index changes with temperature (thermo-optic effect) and length of the optical fiber also changes with temperature (thermal expansion), the phase of the interferometric pattern will consequently change. If $L(T)$ and $\Delta n(T)$ represent the waist length and the difference of indices of two interfering modes at temperature $T$ respectively, then Eq. (2) will take the form

$$
\phi=\frac{2 \pi}{\lambda} \Delta n(T) L(T)
$$

and

$$
\begin{gathered}
\Delta n(T)=\Delta n\left(T_{0}\right)[1+\eta \cdot \Delta T] \\
L(T)=L\left(T_{0}\right)[1+\alpha \cdot \Delta T]
\end{gathered}
$$

where $\Delta n\left(T_{0}\right)=\Delta n$ at room temperature $T_{0}$, $\eta=(\partial \Delta n / \partial T)_{T=T_{0}}$ is the thermo-optic coefficient of silica glass, $L\left(T_{0}\right)$ is the waist length at room temperature $T_{0}$ and $\alpha\left(=5.5 \cdot 10^{-7} \mathrm{~K}^{-1}\right)$ is the thermal expansion coefficient of the silica glass [20].

Eqs. (4a) and (4b) show that variation in temperature changes $\Delta n$ and $L$, which will change the phase of the interferometry intensity accordingly. Consequently, there will be a wavelength shift, $\Delta \lambda$, in the interference spectrum. The expression for relative wavelength shift $\Delta \lambda / \lambda$ caused by a temperature change $\Delta T$ can be derived simply by using Eqs. (3), (4a) and (4b), and can be expressed as

$$
\frac{\Delta \lambda}{\lambda}=-\frac{1}{\Delta n\left(T_{0}\right) L(T)}\left(\frac{\partial \Delta n}{\partial T}\right)_{T=T_{0}} \cdot L\left(T_{0}\right) \Delta T
$$

Eq. (5) establishes a direct relationship between the wavelength shift and the temperature variation with respect to the initial temperature $T_{0}$. The variation of the temperature implies a shift of the pattern with the pattern at initial temperature, which can be evaluated by writing the peak wavelength as a function of the temperature. Similar to our predicted relation in Eq. (5), E. Li et al. have also established the relation for wavelength shift caused by change in temperature in their article and is expressed as [21];

$$
\frac{\Delta \lambda}{\lambda} \approx(\alpha+n) \Delta T
$$

The free spectral range (FSR) is one of the best example of the interference pattern and is defined as the distance between two adjacent peaks/valleys in the spectrum pattern. The expression for FSR is written as [22];

$$
F S R \approx \frac{\lambda^{2}}{\Delta n \cdot L}
$$

From Eq. (7), it is clear that the FSR strongly depends on the change in refractive indices as well as on the waist length. The change in temperature affects the refractive indices of the core and the cladding modes (thermo-optic effect) as well as the fiber length (thermal expansion), thus the interference fringes will shift accordingly.

\section{EXPERIMENTAL SET-UP AND FABRICATION OF TAPERED FIBER}

For the fabrication of tapered fibers, a highly precise, computer-controlled machine having a filament as a heat source, Vytran GPX-3400 is utilized. After stripping off the outer jacket of a single mode fiber of length about $30 \mathrm{~mm}$, the stripped fiber is cleaned with isopropanol alcohol and is then placed on the fiber holding blocks of the Vytran GPX-3400 for the tapering process. In general, a traditional method of heat-and-pull rig for tapering is deployed. The main advantage of this Vytran machine over the traditional method is the uniformity of the waist region. This machine also offers high reproducibility of the tapered fibers and the convenience of changing the design profiles. To ensure the uniformity of the taper fabricated in this experiment, the pulling speed of the fiber holding blocks is kept at a constant rate of $1 \mathrm{~mm} / \mathrm{s}$ while the heat is set at $38 \mathrm{~W}$. 


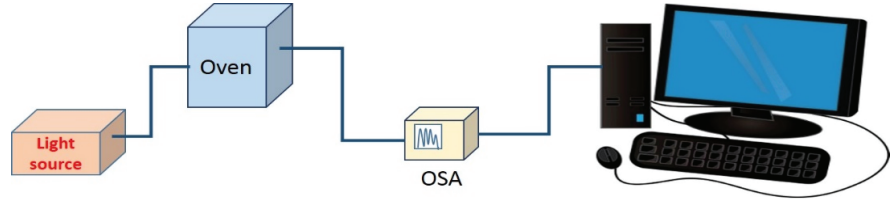

FIG. 2 Schematic diagram of experimental setup for the proposed temperature sensor.

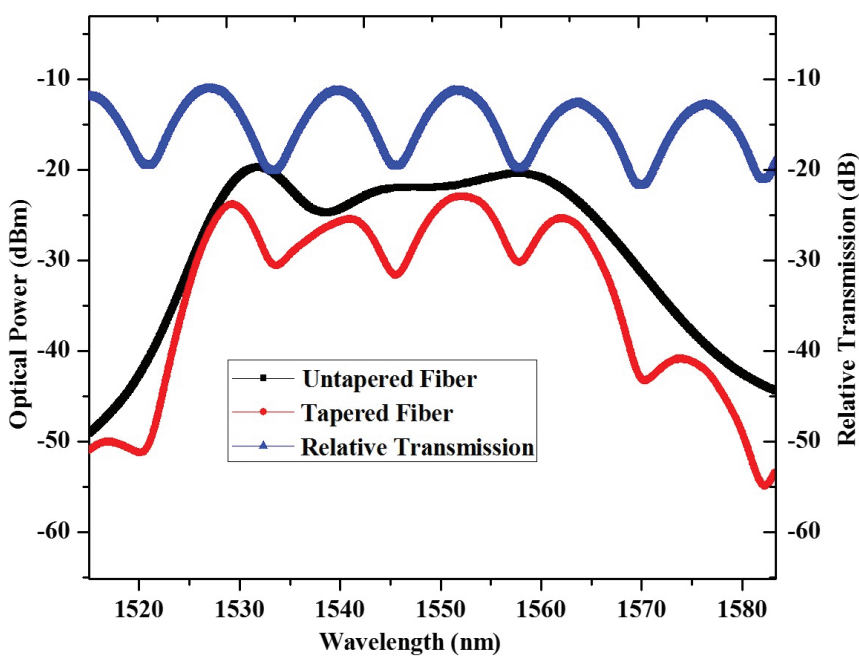

FIG. 3 Transmission spectra for untapered and tapered fibers profile 5-10-5 $\mathrm{mm}$ with waist diameter of $12 \mu \mathrm{m}$.

The experimental setup to study the temperature effect over the transmission spectrum of the SMTF is depicted in Figure 2. The characterization system consists of a broadband light source from 1525 to $1568 \mathrm{~nm}$. Firstly, a reference spectrum is measured by connecting the light source directly to an optical spectrum analyzer (OSA). Then the fabricated SMTF (sensor) is inserted in between the light source and OSA to measure the sensing performance against surrounding temperatures. To create a controlled surrounding temperature, the SMTF is placed in an oven. The oven temperature is varied from $30^{\circ} \mathrm{C}$ to $150^{\circ} \mathrm{C}$ with steps of $5^{\circ} \mathrm{C}$.

\section{RESULTS AND DISCUSSION}

The typical normalized transmission spectrum of the tapered fiber with profile of 5-10-5 mm (10 mm taper length and $5 \mathrm{~mm}$ downtaper/uptaper regions) having waist diameter $12 \mu \mathrm{m}$ is shown in Figure 3, and the transmission spectrum of the untapered fiber is also shown as a reference. From the figure it is clear that, the tapered fiber displays a periodic interferometry pattern that is nonexistence in the spectrum of the untapered fiber. The relative transmission spectrum of tapered fiber with respect to transmission spectrum of untapered fiber is also illustrated in Figure 3. The OSA spectral resolution was set at $0.1 \mathrm{~nm}$ throughout the experiment.

Figure 4(a) shows the transmission spectra of the fabricated optical interferometer based temperature sensor with waist diameter of $12 \mu \mathrm{m}$, which were recorded at different temperature settings $\left(30^{\circ} \mathrm{C}, 50^{\circ} \mathrm{C}, 70^{\circ} \mathrm{C}, 90^{\circ} \mathrm{C}, 110^{\circ} \mathrm{C}, 130^{\circ} \mathrm{C}\right.$ and $\left.150^{\circ} \mathrm{C}\right)$. As theoretically expected, the experimental results show a shift in the peak positions of the transmission spec-
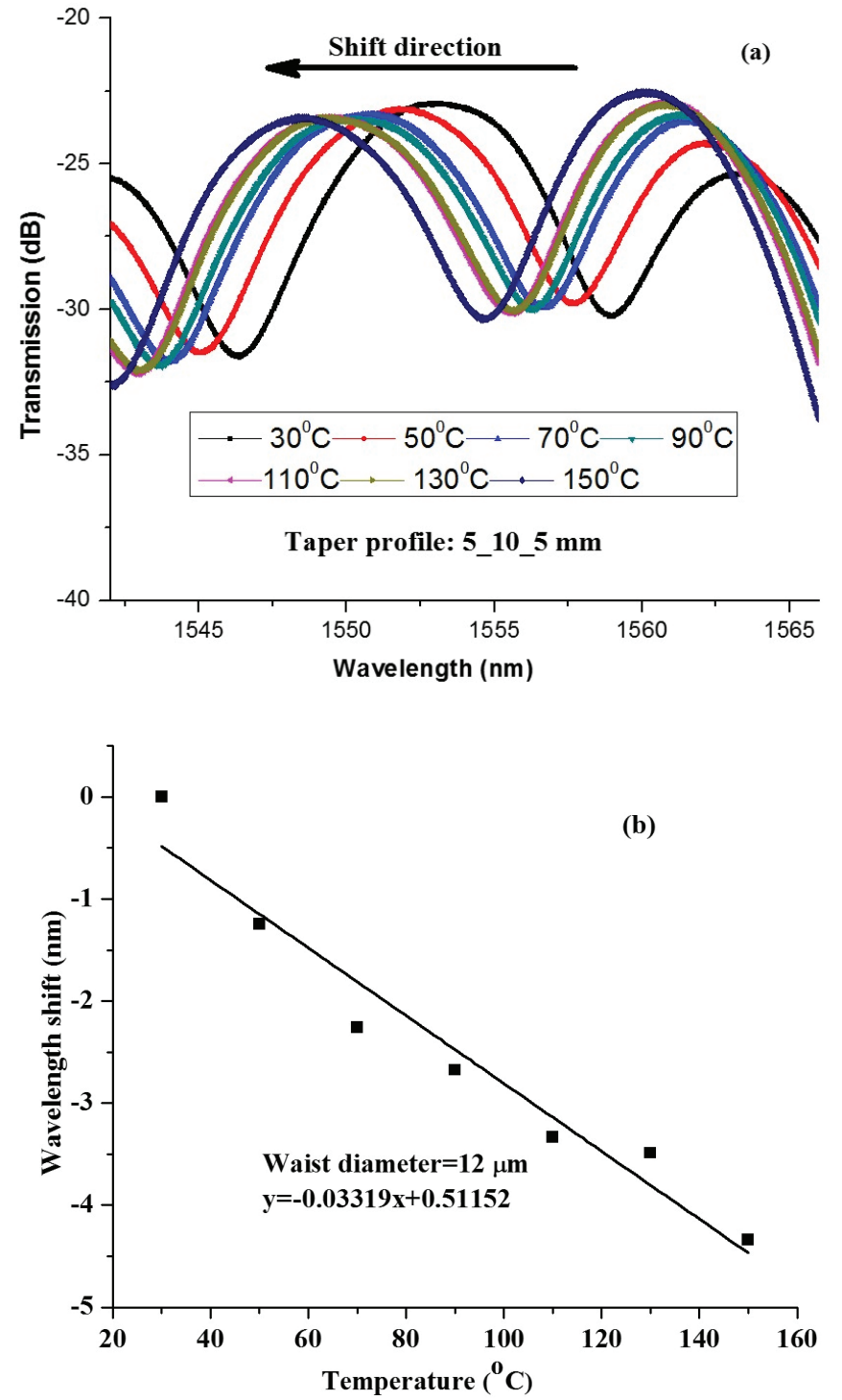

FIG. 4 (a) Transmission spectra and (b) variation of wavelength shift with temperature for taper profile 5-10-5 mm with waist diameter $12 \mu \mathrm{m}$.

trum to a lower wavelength region (blue shift) with respect to the first peak as a consequence of the variation of temperature i.e. negative wavelength shift due to the negative thermo-optic coefficient of the silica glass (core of the fiber). In this case, there are two parameters that characterize the effect of temperature on the transmission spectra of the SMTF; the thermal expansion coefficient and the thermo-optic coefficient in Eqs. (5) and (6) respectively. However, thermo-optic coefficient is the more dominant effect in single-mode optical fiber as compared to thermal expansion coefficient. The plot of the experimental findings of wavelength shift with temperature is illustrated in Figure 4(b). The sensitivity of the fabricated temperature sensor device is also calculated by pitting the change in wavelength shift with respect to temperature change i.e. the gradient of the linear curve plotted wavelength shift against temperature. The sensitivity of the fabricated device with waist diameter $12 \mu \mathrm{m}$ was found to be $0.03319 \mathrm{~nm} /{ }^{\circ} \mathrm{C}$.

To investigate the temperature response with varying taper profile, we fabricated six tapered fibers with different waist 


\begin{tabular}{|c|c|c|c|c|c|}
\hline Sensor ID & $\begin{array}{c}\text { Downtaper } \boldsymbol{a} \\
\mathbf{( \mathbf { m m } )}\end{array}$ & $\begin{array}{c}\text { Uptaper } \boldsymbol{b} \\
\mathbf{( \mathbf { m m } )}\end{array}$ & $\begin{array}{c}\text { Waist length } \boldsymbol{L} \\
\mathbf{( \mathbf { m m } )}\end{array}$ & $\begin{array}{c}\text { Waist diameter } \boldsymbol{d} \\
\mathbf{( \mu \mathbf { m } )}\end{array}$ & $\begin{array}{c}\text { Sensitivity } \\
\left(\mathbf{n m} /{ }^{\circ} \mathbf{C}\right)\end{array}$ \\
\hline $\mathrm{S}_{1}$ & 5 & 5 & 10 & 12 & 0.03319 \\
$\mathrm{~S}_{2}$ & 5 & 5 & 10 & 10 & 0.03406 \\
$\mathrm{~S}_{3}$ & 5 & 5 & 10 & 9 & 0.02149 \\
$\mathrm{~S}_{4}$ & 5 & 5 & 10 & 8 & 0.02673 \\
$\mathrm{~S}_{5}$ & 5 & 5 & 10 & 7 & 0.01143 \\
\hline
\end{tabular}

TABLE 1 Comparative study of sensitivity of different taper profiles with waist length fixed at $L=10 \mathrm{~mm}$.

diameter while keeping the uptaper and downtaper regions constant at $5 \mathrm{~mm}$. The waist length was also fixed at $10 \mathrm{~mm}$. The chosen taper profile of 5-10-5 mm having variation of waist diameters $10,9,8$ and $7 \mu \mathrm{m}$ was tested at different temperatures $\left(30^{\circ} \mathrm{C}, 50^{\circ} \mathrm{C}, 70^{\circ} \mathrm{C}, 90^{\circ} \mathrm{C}, 110^{\circ} \mathrm{C}, 130^{\circ} \mathrm{C}\right.$ and $\left.150^{\circ} \mathrm{C}\right)$. The sensitivity was measured for these profiles from their corresponding wavelength shifts. For the comparative study of the temperature sensors sensitivity, we have collected the sensitivity with various taper profiles in Table 1. From these findings, our results show a decrement of sensitivity with respect to the fiber waist diameter. However, this observation contradicts the effect of wavelength shift predicted in Eq. (5). From thoretical aspect, the fiber waist diameter has no influence on the wavelength shift sensitivity. Although the waist diameter does not contribute directly in the modes coupling, it plays a big role indirectly because the excitement of the higher order modes depends on the V-number. In this case, the V-number is the function of waist diameter. If the waist diameter is larger, more cladding modes will be excited and thus, interferometric pattern will be less pronounced i.e., transmission spectra due to coupling of these modes with fundamental core mode. On the other hand, smaller waist diameters decrease the $\mathrm{V}$ number thus reducing the number of excited cladding modes, resulting in a clear interferometric pattern. Therefore, the shift in peak or dips of transmission spectra can be easily justified. From this experiment, the impact of waist diameter must be taken into account to ensure that the sensor performance can be optimized. The sensitivity of the sensor can be further improved by introducing temperature-sensitive or thermo-chromic materials to the taper waist region, which may be considered in our future research work on the temperature sensors.

\section{CONCLUSION}

We have demonstrated the impact of waist diameter in the implementation of single mode tapered optical fiber as a temperature sensor. The sensitivity of the proposed device is found to be good for the tapered profile 5-10-5 mm with waist diameter $10 \mu \mathrm{m}$ and $12 \mu \mathrm{m}$ achieving sensitivity of $0.03406 \mathrm{~nm} /{ }^{\circ} \mathrm{C}$ and $0.03319 \mathrm{~nm} /{ }^{\circ} \mathrm{C}$ respectively. The optimum profile according to our results was achieved with the profile of 5-10-5 mm having waist diameter of $10 \mu \mathrm{m}$. The main advantages of our sensor are their simple fabrication procedures and high reproduction property as well as mechanical reliability compared to the existing sensors based on tapered optical fiber. Despite its simplicity, the temperature sensitivity exhibited by this sensor is comparable with other tapered-fiber based temperature sensors.

\section{ACKNOWLEDGEMENTS}

One of the authors Dr. T. K. Yadav is thankful to Universiti Putra Malaysia for providing postdoctoral research fellowship. We would also like to thank Amirah Abdul Latif and Mas Izyani Md Ali for their technical support in the completion of this work. This work is partly supported by the Ministry of Education, Malaysia and Universiti Putra Malaysia under Research University Grant Scheme, 05-02-12-2015RU.

\section{References}

[1] 0. S. Wolfbeis, "Fiber-optic chemical sensors and biosensors," Anal. Chem. 76, 3269-3284 (2004).

[2] S. W. Harun, K. S. Lim, A. A. Jasim, and H. Ahmad, "Dual wavelength erbium-doped fiber laser using a tapered fiber," J. Mod. Opt. 57, 1362-3044 (2010).

[3] M. Martí-Panameño, L. C. Goméz-Pavón, A. Luis-Ramos, M. M. Méndez-Otero, and M. D. Iturbe Castillo, "Self mode locking action in a dual-core ring fiber laser," Opt. Commun. 194, 409-414 (2001).

[4] A. Mafi, and J. V. Moloney, "Phase locking in a passive multicore photonic crystal fiber," J. Opt. Soc. Am. B 21, 897-902 (2004).

[5] C. H. Lee, J. Lee, M. K. Kim, and K. T. Kim, “Characteristics of a fiber Bragg grating temperature sensor using the thermal strain of an external tube," J. Korean Phys. Soc. 59, 3188-3191 (2011).

[6] K. J. Han, Y. W. Lee, J. Kwon, S. Roh, J. Jung, and B. Lee, “Simultaneous measurement of strain and temperature incorporating a long-period fiber grating inscribed on a polarization-maintaining fiber," IEEE Photonic. Tech. L. 16, 2114-2116 (2004).

[7] Y. Zhan, K. Gu, H. Wu, and J. Luo, "A combined long period fiber grating multi-parameter sensor," Sensor Rev. 33, 220-227 (2013).

[8] G. Coviello, V. Finazzi, J. Villatoro, and V. Pruneri, “Thermally stabilized PCF-based sensor for temperature measurements up to $1000^{\circ}$ C," Opt. Express 17, 21551-21559 (2009).

[9] V. Finazzi, J. Villatoro, G. Coviello, and V. Pruneri, Photonic crystal fibre sensor for high temperature energy environment (optics and Photonics for Advanced Energy Technology, Cambridge, June 24-25, 2009).

[10] A. J. C. Tubb, F. P. Payne, R. B. Millington, and C. R. Lowe, "Singlemode optical fibre surface plasma wave chemical sensor," Sens. Actuators B Chem. 41, 71-79 (1997).

[11] A. Romolini, R. Falciai, and A. Schena, "Biconically-tapered optical fiber probes for the measurement of esophageal pressure," Sens. Actuators A 70, 205-210 (1998).

[12] M. Shadaram, L. Espada, J. Martinez, and F. Garcia, "Modeling and performance evaluation of ferrocene-based polymer clad tapered optical fiber gas sensors," Opt. Eng. 37, 1124-1129 (1998). 
[13] P. N. Moar, S. T. Huntington, J. Katsifolis, L. W. Cahill, A. Roberts, and K. A. Nugent, "Fabrication, modeling, and direct evanescent field measurement of tapered optical fiber sensors," J. Appl. Phys. 85, 3395-3398 (1999).

[14] C. Bariáin, I. R. Matías, F. J. Arregui, and M. López-Amo, “Optical fiber humidity sensor based on a tapered fiber coated with agarose gel," Sens. Actuators B 69, 127-131 (2000).

[15] W. Henry, "Evanescent field devices: a comparison between tapered optical fibres and polished or D-fibres," Opt. Quantum Electron 26, S261-S272 (1994).

[16] B. H. Lee, and J. Nishii, "Dependence of fringe spacing on the grating separation in a long-period fiber grating pair," Appl. Opt. 38, 3450-3459 (1999).

[17] L. Xu, L. Jiang, S. Wang, B. Li, and Y. Lu, “High temperature sensor based on an abrupt-taper Michelson interferometer in single-mode fiber," Appl. Opt. 52, 2038-2041 (2013).
[18] D. Monzón-Hernández, V. P. Minkovich, and J. Villatoro, “Hightemperature sensing with tapers made of microstructured optical fiber," IEEE Photonic. Tech. L. 18, 511-513 (2006).

[19] A. Chatak, Optics (Tata McGraw-Hill, New Delhi, 2009).

[20] M. J. Weber, Handbook of Optical Materials (CRC Press, Boca Raton, 2003).

[21] E. Li, X. Wang, and C. Zhang, "Fiber-optic temperature sensor based on interference of selective higher-order modes," Appl. Phys. Lett. 89, 091119 (2006).

[22] L. Nguyen, D. Hwang, S. Moon, D. Moon, and Y. Chung, "High temperature fiber sensor with high sensitivity based on core diameter mismatch," Opt. Express 16, 11369-11375 (2008). 